\title{
The Roles of Alcohol Use Severity and Posttraumatic Stress Disorder Symptoms as Risk Factors for Women's Intimate Partner Violence Experiences
}

\author{
Colin T. Mahoney, $\mathrm{PhD}^{1,2}$ and Katherine M. Iverson, $\mathrm{PhD}^{2,3}$
}

\begin{abstract}
Background: To reduce women's risk for intimate partner violence (IPV), it is critical to elucidate malleable psychiatric and behavioral health risk factors. We aimed to identify if alcohol use problems moderate the associations between posttraumatic stress disorder (PTSD) symptoms and past-year IPV victimization experiences among female veterans, a population at high risk for IPV.

Methods: Female veterans $(N=198)$ completed mail surveys assessing PTSD symptoms, alcohol use, and IPV at two time points between 2012 and 2013. Regression analyses were used to examine the associations between PTSD symptoms and alcohol use at Time 1 with past-year IPV at Time 2.

Results: More than a third of women (36.9\%) reported past-year IPV. Alcohol use severity significantly moderated the association between PTSD symptoms and past-year physical IPV. Specifically, there was a significant positive association between PTSD symptoms and past-year IPV at high levels of alcohol use severity, but not at low or average levels of alcohol use. Although both PTSD symptoms and alcohol use were independent risk factors for past-year sexual and psychological IPV, no moderating effect was found for these IPV types.

Conclusions: Findings can inform tailored IPV, psychiatric, and behavioral health screening and counseling. Such practices may reduce psychiatric distress and IPV risk.
\end{abstract}

Keywords: women, veterans, intimate partner violence, PTSD symptoms, alcohol use

\section{Introduction}

$\mathbf{I}_{\mathrm{e}}^{\mathrm{N}}$ NTIMATE PARTNER VIOLENCE (IPV) victimization includes experiences of physical, sexual, and psychological aggression by an intimate partner. ${ }^{1}$ This form of violence disproportionately affects women, as approximately one-third of women worldwide who have been in an intimate relationship have experienced one or more forms of IPV. ${ }^{2}$ Approximately one in three women in the United States experience physical IPV in their lifetimes, while one in six women $(16.4 \%)$ experience some form of sexual IPV (e.g., rape or unwanted sexual contact), and nearly half $(47.1 \%)$ experience psychological IPV. ${ }^{3}$

\section{IPV in female veterans}

Women who have served in the military are a population at high risk for experiencing IPV. ${ }^{4-7}$ One study using a national survey of female veterans found that $55 \%$ experienced IPV during their lifetime, with the majority of these women experiencing multiple forms of abuse. ${ }^{8}$ Approximately one in five female Veterans Health Administration (VHA) patients experience past-year IPV, which is associated with high primary care utilization. ${ }^{9}$ While using VHA's adopted IPV screening instrument, the Hurt, Insult, Threaten, and Scream (HITS) tool, ${ }^{10}$ another study found that $\sim 37 \%$ of partnered female veterans screened positive for past-year IPV. ${ }^{11} \mathrm{Wo}$ men who screened positive for IPV were three times more likely to experience depression, two times more likely to experience posttraumatic stress disorder (PTSD), and almost four times more likely to have mental health multimorbidity than women without IPV. ${ }^{11}$

Thus, beyond the high prevalence of IPV in this population, IPV is also associated with a wide range of negative physical and mental health sequelae. Somatic disorders, chronic pain, gynecological issues, sexually transmitted

\footnotetext{
${ }^{1}$ Behavioral Science Division, National Center for PTSD, VA Boston Healthcare System, Boston, Massachusetts.

${ }^{2}$ Women's Health Sciences Division, National Center for PTSD, VA Boston Healthcare System, Boston, Massachusetts.

${ }^{3}$ Department of Psychiatry, Boston University School of Medicine, Boston, Massachusetts.
} 
infections, anxiety, suicidality, nonsuicidal self-injury, and sleep disorders are caused or exacerbated by IPV. ${ }^{12}$ IPV is strongly associated with a range of psychiatric symptoms among female veterans, even when adjusting for the strong effects of military sexual trauma (MST; i.e., sexual harassment and sexual assault during military service) and combat exposure. ${ }^{7,13}$ Given the deleterious effects on the lives and health of women, there is a need to elucidate factors that can be targeted during assessment and treatment, especially those that IPV survivors can employ to lower risk of future violence. ${ }^{14,15}$ Providers, especially those working in mental health and primary care contexts, could benefit from research that can help them identify and intervene with those most vulnerable to experiencing IPV.

\section{Risk factors for IPV}

Prior IPV and interpersonal violence experiences elevate risk for future IPV. ${ }^{16,17}$ In a sample of 724 community women, Testa et al. found that a lifetime history of psychological and physical IPV predicted physical violence 12 months later from the same partner. ${ }^{18}$ Another study found that greater cumulative interpersonal victimization predicted IPV from a new partner 12 months later in a large sample $(N=756)$ of community women who recently obtained protective orders. ${ }^{19}$ Women are also five times more likely to experience physical or emotional IPV if they experienced childhood physical or emotional abuse in comparison with women without this history. ${ }^{20,21}$ Furthermore, women with a history of childhood sexual abuse (CSA) are $\sim 25$ times more likely to experience IPV in more than one relationship as an adult, ${ }^{22}$ consistent with previous literature demonstrating CSA as a risk factor for IPV. ${ }^{23-26}$

In terms of informing care in integrated primary care and mental health settings, there is a growing literature identifying psychiatric and behavioral health symptoms and conditions as possible risk factors. In particular, posttraumatic psychopathology, although frequently a consequence of experiencing partner violence, ${ }^{27,28}$ when left untreated, can increase women's risk for future IPV. In a longitudinal survey study, PTSD symptom severity predicted IPV revictimization above and beyond severity of past IPV. ${ }^{29}$ Furthermore, PTSD hyperarousal symptoms also significantly predicted physical IPV revictimization in women who sought shelter from IPV. ${ }^{30}$ This increased risk is also apparent in treatment studies; women with a history of interpersonal trauma (including IPV) who engaged in cognitive behavioral therapy for PTSD and experienced large decreases in PTSD symptoms were significantly less likely to report IPV 6 months later in comparison with women without improvement in PTSD. ${ }^{15}$

The pathway from PTSD to IPV, however, is unclear. One potential factor may be alcohol use; individuals with PTSD are at heightened risk of concurrent alcohol use. ${ }^{31-34}$ Individuals with PTSD theoretically use substances to reduce negative emotionality associated with posttraumatic distress, consistent with the self-medication hypothesis. ${ }^{35-37}$ For women with a history of IPV, alcohol is likely being used to reduce negative affect, PTSD symptoms, and other detrimental outcomes. ${ }^{38-41}$ As a result, alcohol use may increase among female IPV survivors because of its negative reinforcing qualities (i.e., short-term reductions in psycholog- ical pain). These associations between alcohol misuse and IPV have been documented in cross-sectional studies of female veterans. ${ }^{7,11,42}$ For example, one study found that PTSD symptom severity and past-year psychological IPV were positively associated with alcohol misuse in a sample of female veterans; however, in follow-up multivariate analyses, only past-year psychological IPV significantly contributed to alcohol misuse. ${ }^{42}$

Although alcohol use has been studied within the context of PTSD and IPV, the literature is limited regarding alcohol use as a longitudinal risk factor for IPV experiences. One longitudinal study found that women with a history of IPV and PTSD were almost 15 times more likely to use drugs and alcohol over a period of 21 days than women without PTSD, but they did not examine if substance use elevates risk for future IPV. ${ }^{43}$ Another study demonstrated that quantity of alcohol use and PTSD predicted IPV in a large sample of 25,778 community members, but this was a cross-sectional study, PTSD was examined dichotomously (i.e., present or absent), and the interaction of these predictors was not examined. ${ }^{44}$ Other studies have provided evidence for PTSD as a risk factor for IPV experiences as a whole, ${ }^{15,29}$ but no study to date has examined it as a risk factor for various types of IPV (i.e., physical, sexual, and psychological).

Furthermore, the predictive nature of alcohol use and PTSD symptoms on experiences of IPV may be due to diminished threat detection for women; these factors can reduce the ability to determine overt and covert threats of violence. ${ }^{4-47}$ High levels of psychiatric distress such as severe PTSD symptoms, combined with alcohol use to cope with these symptoms and their effects, can compromise threat detection abilities and also complicate decisions and actions necessary to leave abusive relationships. As these factors are critically important for understanding the risk of future IPV experiences, continued research in these domains is needed.

\section{Present study}

There is consequently a gap in current conceptualizations of the role of alcohol use in the association between PTSD symptoms and IPV experiences among women. In addition, although female veterans experience IPV at high rates, ${ }^{6}$ there has been minimal attention to the role of alcohol use in possibly contributing to IPV risk in this population. Therefore, the purpose of the present study is to explore the influence of alcohol use severity on the conditional effect of PTSD symptoms on different forms of IPV experiences (i.e., physical, sexual, and psychological) in a longitudinal cohort of female veterans who completed surveys at baseline (Time 1; T1) and 12 months later (Time 2; T2). We do not expect a significant indirect effect of alcohol use severity on the association between PTSD symptoms and different forms of IPV experiences as PTSD symptoms appear to have a powerful direct effect on the likelihood of experiencing IPV. ${ }^{15,29,30}$ Based on prior research, we hypothesized the following: (1) PTSD symptom severity and alcohol use severity at T1 would significantly predict IPV experiences at T2; (2) alcohol use severity would not mediate the longitudinal relationship between PTSD symptom severity at T1 and IPV experiences at T2; (3) alcohol use severity would moderate the longitudinal association between PTSD symptom severity at T1 and IPV experiences at T2; and (4) specifically 
women who experience PTSD symptoms and average to high severity of alcohol use would be at significantly higher risk of future IPV than women with PTSD symptoms and low severity of alcohol use.

\section{Methods}

\section{Study design and participants}

This was a study of female veterans who received VHA care in the New England region of the United States, randomly selected from the roster of all female VHA patients in the geographic region, were at least 18 years of age, had participated in a larger study ${ }^{48,49}$ that aimed to evaluate the accuracy of a brief screening tool for IPV victimization experiences, and had agreed to be recontacted. Inclusion criteria included identifying as female, at least 18 years of age, and VA users within the previous 2 years. Participants were excluded from the study if they did not meet these criteria. Among the 369 women who participated in the larger study, $70.7 \%(n=261)$ agreed to be recontacted. Of these women, 248 with locatable addresses received a survey and 198 responded ( $79.8 \%$ response rate). There were no differences in demographic characteristics of the 198 women in this substudy compared with those in the larger roster of randomly selected women, nor were there differences in demographic variables between responders and nonresponders.

Participants were between the ages of 23 and 89 years $(\mathrm{M}=51.01$, standard deviation $[\mathrm{SD}]=16.93)$. The sample predominately identified as White $(n=161,77.8 \%)$; of the remaining participants, 18 women identified as Black/African American (9.1\%), 9 identified as American Indian/Alaskan Native (4.6\%), 7 identified as Hispanic/Latina/Spanish origin $(3.5 \%), 1$ identified as Native Hawaiian/Pacific Islander $(0.5 \%)$, and 1 identified as Asian $(0.5 \%)$. Nearly a third of the sample reported being married $(n=60,30.9 \%), 46$ reported being divorced $(23.7 \%), 14(7.2 \%)$ reported living with their partner, 25 (12.9\%) reported being widowed, 5 $(2.5 \%)$ reported being separated, and 44 reported being single/never married $(22.7 \%)$. Approximately one-fifth of the sample reported a household income of $\$ 15,000$ or less $(n=32,17.1 \%), 40$ reported an income between $\$ 15,001$ and $\$ 25,000(21.4 \%), 34$ reported an income between $\$ 25,001$ and $\$ 35,000(18.2 \%), 34$ reported an income between $\$ 35,001$ and $\$ 50,000$ (18.2\%), 22 reported an income between $\$ 50,001$ and $\$ 75,000(11.8 \%), 16$ reported an income between $\$ 75,001$ and $\$ 100,000(8.6 \%)$, and 11 reported an income over $\$ 100,000(5.9 \%)$. Nearly half of the sample had served in the Army $(n=89,46.1 \%)$ with the next highest branch being the Air Force $(n=49,25.4 \%)$, followed by the Navy $(n=39,20.2 \%)$, Marines $(n=13,6.7 \%)$, and Coast Guard $(n=3,1.6 \%)$.

\section{Procedure}

As described previously, ${ }^{48}$ T1 surveys were mailed in 2012 using a modified multiple mailing strategy ${ }^{50}$ to potential participants. The procedures for the survey were as follows: (1) informed consent fact sheet, survey packet, and \$10 incentive; (2) a thank you/reminder postcard 2 weeks later; and (3) an additional survey packet and another $\$ 10$ incentive 4 weeks later to women who had not yet responded. This same strategy was employed 12 months later for the T2 survey. The
T1 and T2 survey administration occurred during an approximate 3-month interval. The local VA institutional review board approved study procedures.

\section{Measures}

Trauma history. MST was determined using two items from the Trauma Questionnaire ${ }^{51}$ at T1: "While you were in the military, did you receive uninvited and unwanted sexual attention, such as touching or cornering, pressure for sexual favors, or verbal remarks?" and "While you were in the military, did someone ever use force or the threat of force to have sexual contact with you?' A positive endorsement of either item signified MST (yes/no).

CSA was determined using the Trauma History Screen (THS) at T1, a 13-item measure of exposure to traumatic events. ${ }^{52}$ Participants are prompted to indicate whether a trauma occurred (yes/no). The THS has demonstrated good psychometric properties with evidence of construct and convergent validity as well as test-retest reliability. ${ }^{52}$

Psychiatric and behavioral health symptoms. PTSD symptom severity was assessed at T1 using the PTSD Checklist (PCL), a 17-item measure of DSM-IV PTSD symptoms. ${ }^{53}$ Participants rate the degree to which each symptom bothered them on a 5 -point scale ( $1=$ not at all to $5=$ extremely), resulting in total scores ranging from 17 to 85 . The PCL has demonstrated strong psychometric properties, with evidence of high internal consistency $(\alpha=0.95)$, good test-retest reliability $(r=0.68-0.96)$, and good convergent and discriminant validity. ${ }^{54-57}$ The PCL demonstrated strong internal consistency in the present study $(\alpha=0.96)$. We converted PCL scores to PTSD Checklist for DSM-5 (PCL-5) ${ }^{58}$ scores using score conversions from a previously validated crosswalk procedure ${ }^{59}$ and used the recommended cutoff score of 33 to determine probable PTSD status. ${ }^{60,61}$ The crosswalk procedure was computed using equipercentile equating with log-linear smoothing in a sample of 1003 US veterans oversampled for PTSD who completed both the PCL and PCL-5; evaluations of the concordance between the crosswalk determined that predicted PCL-5 scores and observed PCL-5 scores were highly congruent (Intraclass Correlation Coefficient, ICC $=0.96$ ), with no differences in accuracy by demographic factors (e.g., age and gender) or PTSD status. ${ }^{59}$ This procedure was conducted in the present study to ensure that symptoms reflect current conceptualizations of PTSD symptomatology.

Overall levels of severity for alcohol use/misuse at T1 were determined using the 10-item Alcohol Use Disorders Identification Test (AUDIT). ${ }^{62}$ Scores range from 0 to 40 , with a score of 6 or more for females indicating hazardous alcohol consumption and possible alcohol dependence. ${ }^{63}$ The AUDIT has demonstrated strong psychometric properties with evidence of convergent validity, strong test-retest reliability $(r=0.84-0.95)$, and good internal consistency $(\alpha=0.81) .{ }^{64-67}$ In the present study, the AUDIT demonstrated good internal consistency $(\alpha=0.81)$.

Intimate partner violence. IPV experiences were assessed at T1 and T2 with the Conflict Tactics Scales, Revised (CTS-2), a well-established 78-item measure of relational conflict, including physical, sexual, and psychological IPV experiences. ${ }^{68}$ Each item prompts participants to indicate how frequently they have experienced each behavior over the 
past year on a 7-point scale (i.e., $0=$ never to $6=$ more than 20 times). The $\mathrm{T} 1$ administration also prompted participants to indicate whether they had experienced each behavior before the past year. We used the 12-item physical IPV subscale, the 7-item sexual IPV subscale, and the 8-item psychological IPV subscale. The CTS-2 has demonstrated strong psychometric properties across populations, including good construct, convergent, and discriminant validity, along with good reliability. ${ }^{69-71}$

For the present study, scores for physical, sexual, and psychological IPV were computed by summing the number of items that were positively endorsed for each IPV subscale. This variety score method has good psychometric properties, reduces errors of estimation that occur during recall of highor low-frequency events, and does not require weighting acts by their severity. ${ }^{72}$ The CTS- 2 subscales demonstrated adequate to strong internal consistency estimates (lifetime physical $[\alpha=0.94]$, sexual $[\alpha=0.86]$, and psychological $[\alpha=0.90]$ IPV at T1; past-year physical $[\alpha=0.82]$, sexual $[\alpha=0.75]$, and psychological $[\alpha=0.81]$ IPV at T2).

Demographics. Participants completed a questionnaire in which they reported age, ethnicity, race, highest level of education, current marital status, children, living situation, employment status, household income, years of military service, military deployments, branch of the military, and military rank.

\section{Data analysis}

Pearson correlations were first computed to examine bivariate associations among demographic characteristics, PTSD symptom severity, severity of alcohol use, and the different forms of IPV (i.e., lifetime IPV at T1 and past-year IPV at T2). Mediation and moderation analyses were then conducted using regression analyses in the PROCESS macro for IBM SPSS, version $25,{ }^{73}$ controlling for age, Army service branch (yes/no), non-White race (yes/no), MST (yes/no), CSA (yes/no), and lifetime physical, sexual, and psychological IPV at T1, as previous studies have established these variables as risk factors for IPV. ${ }^{11,24,48}$ Alcohol use mediates the association between PTSD symptom severity and past-year IPV experiences if the indirect effect of alcohol use is significant, suggesting that the effect of PTSD symptoms operates through alcohol use in increasing risk for future IPV. The PROCESS macro in SPSS provides asymmetric confidence intervals to determine if the indirect effect is significant.

The effect of PTSD symptoms on past-year IPV experiences is moderated by alcohol use severity if the sign, size, or strength of the relationship between PTSD and IPV is contingent upon or predicted by alcohol use. ${ }^{73}$ PTSD symptoms and alcohol use severity thus would need to exhibit a significant interaction in their influence on past-year IPV experiences for alcohol use to be considered a significant moderator of their association. The alcohol use severity $\times$ PTSD symptom severity interaction was plotted at the 16th percentile (i.e., low), 50th percentile (i.e., average), and 84th percentile (i.e., high) for alcohol use across low (i.e., 1 SD below the mean), average (i.e., at the mean), and high (i.e., $1 \mathrm{SD}$ above the mean) levels of PTSD symptoms. This statistical approach was consistent with established moderation procedures. ${ }^{73}$ The PROCESS macro in SPSS provides output surrounding the conditional effects of PTSD symptoms at each level of alcohol use along with data to visualize the interaction of these two variables. ${ }^{73}$ It also provides asymmetric confidence intervals to determine if the size, sign, or strength of the relationship between PTSD and IPV differs between each value of alcohol use severity. Within the data set, 11 participants had missing data on one or more of the present study's primary measures; they were subsequently excluded from analyses.

\section{Results}

At T1, PTSD total symptom severity scores ranged from 0 to $80(M=20.68, S D=20.29)$, with $28.8 \%(n=57)$ meeting criteria for probable PTSD. Alcohol use scores ranged from 0 to 28 and indicated some problematic drinking on average $(\mathrm{M}=2.76, \mathrm{SD}=4.19)$. At $\mathrm{T} 2$, over a third of women $(36.9 \%)$ experienced past-year physical, sexual, and/or psychological IPV. See Table 1 for descriptive statistics of the study variables and covariates, including the proportion of women who reported each type of past-year IPV. Table 2 displays bivariate correlations between study variables. PTSD symptom severity was significantly related to alcohol use severity and all forms of IPV at both time points. However, alcohol use severity was significantly correlated with physical IPV and psychological IPV at T1 and T2, but not sexual IPV at either time point.

\section{Primary analyses}

In terms of hypothesis 1 , the regression analyses indicated that alcohol use severity and PTSD symptom severity at T1 significantly predicted physical IPV at T2 (Table 3). For sexual and psychological IPV, the only covariate that significantly predicted past-year IPV at T2 was a lifetime history of either sexual or psychological IPV at T1.

For hypothesis 2 , the mediation analyses demonstrated that there were no significant indirect effects of PTSD symptom severity on past-year physical IPV $(-0.04$ to 0.18$)$, past-year sexual IPV ( -0.03 to 0.07$)$, and past-year psychological IPV $(-0.04$ to 0.04$)$ experiences at T2 via alcohol use at T1. Therefore, alcohol use severity does not significantly mediate the relationships between PTSD symptom severity and pastyear IPV experiences in the present study.

With regard to hypothesis 3, the moderation analyses for physical IPV revealed that an interaction term of PTSD symptom severity and alcohol use severity was significantly associated with physical IPV experiences at T2, $F(11$, $116)=16.04, p<0.001$, and $R^{2}=0.69$ (Table 3). Alcohol use, PTSD symptoms, non-White race, lifetime physical IPV, and lifetime sexual IPV were all significant predictors of pastyear physical IPV. The plotted interaction of alcohol use severity and PTSD symptoms (Fig. 1) demonstrates that women with the highest levels of PTSD symptoms and the highest severity of alcohol use reported significantly more acts of past-year physical IPV at T2, providing some evidence for hypothesis 3. However, no significant effect of PTSD symptoms on acts of past-year physical IPV was found for women with low to average alcohol use, indicating that PTSD symptom severity is not a significant risk factor for past-year physical IPV at these levels of alcohol use severity.

Interaction terms consisting of PTSD symptom severity and alcohol use severity were not significantly associated 
Table 1. Descriptive Statistics of Primary Study Variables and Covariates at Time 1 and Time $2(N=198)$

\begin{tabular}{|c|c|c|c|c|c|c|}
\hline \multirow[b]{2}{*}{ Variable } & \multicolumn{3}{|c|}{$T 1$} & \multicolumn{3}{|c|}{$T 2$} \\
\hline & $M(S D)$ & Range & $\mathrm{n}(\%)$ & $M(S D)$ & Range & $\mathrm{n}(\%)$ \\
\hline Age & $51.01(16.93)$ & $23-89$ & & & & \\
\hline Army+ & & & 89 (44.9) & & & \\
\hline Non-White race+ & & & $37(18.7)$ & & & \\
\hline MST+ & & & $108(54.5)$ & & & \\
\hline $\mathrm{CSA}+$ & & & $50(25.3)$ & & & \\
\hline PTSD symptoms & $20.68(20.29)$ & $0-80$ & & & & \\
\hline AU severity & $2.76(4.19)$ & $0-28$ & & & & \\
\hline Physical IPV & $0.20(0.68)$ & $0-4$ & $30(15.15)$ & $0.17(0.81)$ & $0-7$ & $15(7.58)$ \\
\hline Sexual IPV & $0.17(0.57)$ & $0-4$ & $11(5.56)$ & $0.14(0.53)$ & $0-4$ & $17(8.59)$ \\
\hline Psychological IPV & $1.77(1.81)$ & $0-7$ & $63(31.82)$ & $0.93(1.55)$ & $0-7$ & $71(35.86)$ \\
\hline
\end{tabular}

AU, alcohol use; CSA, childhood sexual abuse; IPV, intimate partner violence; MST, military sexual trauma; PTSD, posttraumatic stress disorder; SD, standard deviation; T1, Time 1; T2, Time 2 (12-month follow-up) $+=$ dichotomous variable.

with past-year sexual or psychological IPV at T2 (Table 3). Thus, alcohol use severity does not significantly moderate the associations between PTSD symptom severity and nonphysical forms of IPV in the present study. Severity of alcohol use and PTSD symptom severity also were not significant independent predictors of past-year sexual and psychological IPV (Table 3).

\section{Discussion}

The present study investigated the role of alcohol use severity and PTSD symptoms as risk factors for IPV experiences in a sample of female veterans. Our first hypothesis was partially supported, in that PTSD symptoms and alcohol use at T1 significantly predicted past-year physical IPV experiences at T2 when adjusting for other sociodemographic and interpersonal violence factors known to be associated with IPV risk among female veterans (i.e., age, service branch, non-White race, CSA, and MST). Our second hypothesis was confirmed, in that alcohol use severity did not mediate the relationship between PTSD symptom severity and any of the forms of past-year IPV experiences. Our third hypothesis was also partially supported, in that alcohol use significantly moderated the association between PTSD symptoms and past-year physical IPV at T2. However, PTSD symptom severity and alcohol use severity at T1 were not independent predictors of past-year sexual and psychological forms of IPV at T2, and we did not find a moderating effect of alcohol use for either of these forms of IPV.

The present study's findings with physical IPV were consistent with the extant literature surrounding connections between IPV, PTSD symptoms, and alcohol use. ${ }^{43,44}$ However, this is the first study to date to use a longitudinal design or to conduct moderation analyses to demonstrate the conditional effect of PTSD symptoms due to alcohol use on physical IPV experiences. These findings suggest that women with PTSD symptoms engaging in high amounts of alcohol use are at heightened risk of future physical IPV experiences. Interestingly, if women with PTSD symptoms had low to average severity of alcohol use problems, they were no longer at significant risk for experiences of physical IPV. Therefore, it appears that PTSD symptoms are only a risk factor for physical IPV if women are concurrently experiencing high severity of alcohol use problems. Conceptually, it is possible that women are coping with PTSD symptoms through the use of alcohol (i.e., self-medication hypothesis), ${ }^{35-37}$ considering evidence that veterans with PTSD are four times more likely to have an alcohol use disorder than veterans without PTSD. ${ }^{74}$ They are subsequently at heightened risk, due to this combined vulnerability, of being in relationships that involve alcohol use and violence. In fact, $\sim 15 \%$ of IPV cases involve alcohol use by both partners. ${ }^{44}$ Furthermore, binge drinkers in particular are at elevated risk of experiencing IPV. ${ }^{75,76}$

Table 2. Zero-Order Correlations Among Study Variables $(N=198)$

\begin{tabular}{|c|c|c|c|c|c|c|c|c|}
\hline & PTSD symptoms & $A U$ severity & Phys T1 & Phys T2 & Sex $T 1$ & Sex $T 2$ & Psych T1 & Psych T2 \\
\hline \multicolumn{9}{|l|}{ PTSD symptoms } \\
\hline Alcohol use severity & $0.19^{\mathrm{a}}$ & & & & & & & \\
\hline Phys T1 & $0.31^{b}$ & $0.22^{\mathrm{b}}$ & & & & & & \\
\hline Phys T2 & $0.30^{\mathrm{b}}$ & $0.24^{b}$ & $0.41^{b}$ & & & & & \\
\hline Sex T1 & $0.21^{\mathrm{a}}$ & 0.02 & 0.17 & $0.34^{\mathrm{b}}$ & & & & \\
\hline Sex T2 & $0.31^{b}$ & 0.10 & 0.10 & $0.66^{b}$ & $0.43^{b}$ & & & \\
\hline Psych T1 & $0.33^{b}$ & $0.27^{\mathrm{b}}$ & $0.46^{b}$ & $0.34^{b}$ & $0.36^{b}$ & 0.19 & & \\
\hline Psych T2 & $0.20^{b}$ & $0.16^{\mathrm{a}}$ & $0.26^{b}$ & $0.48^{b}$ & 0.16 & $0.47^{\mathrm{b}}$ & $0.56^{\mathrm{b}}$ & \\
\hline
\end{tabular}

${ }^{\mathrm{a}} p<0.05 ;{ }^{\mathrm{b}} p<0.01$. Bolded values are statistically significant correlation coefficients.

Phys T1, lifetime physical IPV at T1; Phys T2, past-year physical IPV at T2; Psych T1, lifetime psychological IPV at T1; Psych T2, pastyear psychological IPV at T2; Sex T1, lifetime sexual IPV at T1; Sex T2, past-year sexual IPV at T2. 
Table 3. Summary of Multivariate Regression Analyses Predicting Past-Year Intimate Partner Violence EXPERIENCES AT 12-MONTH FOLLOW-Up $(N=198)$

\begin{tabular}{|c|c|c|c|c|}
\hline Variable & $\beta$ & $S E$ & $\mathrm{t}$ & $95 \% C I$ \\
\hline \multicolumn{5}{|l|}{ DV: Physical IPV T2 } \\
\hline Age & -0.001 & 0.004 & -0.35 & -0.01 to 0.01 \\
\hline Army & 0.16 & 0.12 & 1.37 & -0.07 to 0.40 \\
\hline Ethnicity & $0.29^{\mathrm{a}}$ & 0.14 & 2.05 & 0.01 to 0.58 \\
\hline MST & 0.13 & 0.13 & 1.04 & -0.12 to 0.39 \\
\hline CSA & 0.10 & 0.14 & 0.69 & -0.18 to 0.37 \\
\hline L-Physical IPV T1 & $0.28^{\mathrm{b}}$ & 0.09 & 3.05 & 0.10 to 0.47 \\
\hline L-Sexual IPV T1 & $0.62^{\mathrm{c}}$ & 0.14 & 4.32 & 0.33 to 0.90 \\
\hline L-Psych IPV T1 & -0.01 & 0.04 & -0.18 & -0.08 to 0.07 \\
\hline PTSD T1 & $0.04^{\mathrm{a}}$ & 0.01 & 2.11 & 0.01 to 0.08 \\
\hline AU T1 & $0.05^{\mathrm{a}}$ & 0.02 & 2.36 & 0.01 to 0.10 \\
\hline PTSD T1×AU T1 & $0.03^{\mathrm{c}}$ & 0.01 & 6.35 & 0.02 to 0.04 \\
\hline \multicolumn{5}{|l|}{ DV: Sexual IPV T2 } \\
\hline Age & 0.003 & 0.004 & 0.80 & -0.004 to 0.01 \\
\hline Army & 0.03 & 0.11 & 0.22 & -0.20 to 0.25 \\
\hline Ethnicity & 0.21 & 0.14 & 1.57 & -0.06 to 0.49 \\
\hline MST & -0.05 & 0.12 & -0.45 & -0.30 to 0.19 \\
\hline CSA & -0.07 & 0.13 & -0.53 & -0.33 to 0.19 \\
\hline L-Physical IPV T1 & -0.02 & 0.09 & -0.18 & -0.19 to 0.16 \\
\hline L-Sexual IPV T1 & $0.80^{\mathrm{c}}$ & 0.14 & 5.83 & 0.52 to 1.07 \\
\hline L-Psych IPV T1 & -0.03 & 0.04 & -0.72 & -0.10 to 0.05 \\
\hline PTSD T1 & 0.01 & 0.03 & 1.73 & -0.001 to 0.01 \\
\hline AU T1 & 0.01 & 0.02 & 0.02 & -0.04 to 0.04 \\
\hline PTSD T1×AU T1 & 0.003 & 0.001 & 0.69 & -0.001 to 0.001 \\
\hline \multicolumn{5}{|l|}{ DV: Psych IPV T2 } \\
\hline Age & -0.001 & 0.01 & -0.09 & -0.02 to 0.02 \\
\hline Army & 0.53 & 0.35 & 1.52 & -0.16 to 1.23 \\
\hline Ethnicity & 0.53 & 0.42 & 1.37 & -0.26 to 1.41 \\
\hline MST & -0.06 & 0.38 & -0.15 & -0.80 to 0.69 \\
\hline CSA & 0.36 & 0.41 & 0.89 & -0.44 to 1.17 \\
\hline L-Physical IPV T1 & 0.08 & 0.27 & 0.31 & -0.46 to 0.63 \\
\hline L-Sexual IPV T1 & 0.34 & 0.42 & 0.81 & -0.49 to 1.18 \\
\hline L-Psych IPV T1 & $0.48^{\mathrm{c}}$ & 0.11 & 4.38 & 0.26 to 0.70 \\
\hline PTSD T1 & 0.04 & 0.01 & 0.35 & -0.02 to 0.03 \\
\hline AU T1 & 0.03 & 0.07 & 0.39 & -0.10 to 0.16 \\
\hline PTSD T1×AU T1 & 0.004 & 0.002 & -0.29 & -0.003 to 0.003 \\
\hline
\end{tabular}

${ }^{\mathrm{a}} p<0.05 ;{ }^{\mathrm{b}} p<0.01 ;{ }^{\mathrm{c}} p<0.001$.

CI, confidence interval; DV, dependent variable; L-Physical IPV T1, lifetime physical IPV; L-Psych IPV T1, lifetime psychological IPV; L-Sexual IPV T1, lifetime sexual IPV; Physical IPV T2, past-year physical IPV; Psych IPV T2, past-year psychological IPV; SE, standard error; Sexual IPV T2, past-year sexual IPV; T2, Time 2 (12-month follow-up).

FIG. 1. The AU $\times$ total PTSD symptom severity interaction plot of the conditional effect of PTSD symptoms across levels of AU on T2 physical IPV victimization $(N=198)$. AU, alcohol use levels at T1; IPV, intimate partner violence; Physical IPV Count, past-year physical IPV acts at T2; PTSD, posttraumatic stress disorder levels at T1; T1, Time 1; T2, Time 2.

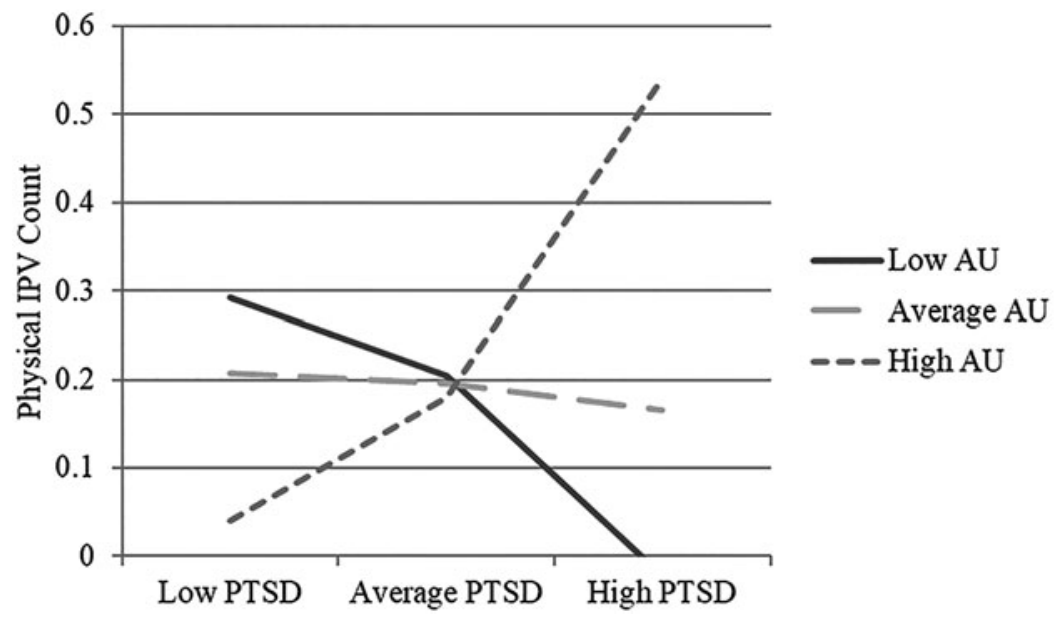


This heightened risk of being in relationships that involve IPV may be due to the interaction of PTSD symptoms and alcohol use in reducing threat detection for women as IPV exposure and both forms of psychopathology can impair the ability to discern the threat of violence. ${ }^{45-47} \mathrm{Al}-$ though violent partners are wholly responsible for their own use of physical IPV, it is possible that women are better able to recognize and respond to signs of a partner becoming violent or aggressive when using lower amounts of alcohol. For example, she may be able to engage in safetypromoting behaviors (i.e., staying in visible places, enlisting others, and moving conversations away from risky topics) or other forms of de-escalation. Hyperarousal and emotional numbing symptoms significantly predict alcohol misuse in veterans while controlling for age, sex, branch of service, combat exposure, and depression status. ${ }^{77}$ Thus, high levels of PTSD symptoms, such as hyperarousal or restricted affect, paired with severe alcohol use may overwhelm one's internal resources and make it more challenging to engage in safety and harm reduction strategies. This hypothesis and other possible explanations should be examined in subsequent mixed-method studies to inform prevention and clinical interventions.

However, it is still unclear why alcohol use would not exhibit this same moderating effect with other forms of IPV. Alcohol use and PTSD symptoms, such as hyperarousal and emotional numbing, may lead to more physical altercations between women and their intimate partners, ${ }^{45-47}$ whereas other factors may be more influential on the risk of future experiences of sexual or psychological IPV, such as characteristics of the individuals that use violence ${ }^{78}$ and/or the use of other substances by survivors. ${ }^{79}$ Future studies should examine the interaction of these variables with PTSD symptoms to determine other factors influencing these types of IPV experiences. Based on our correlation analyses, findings also indicate that PTSD symptoms and alcohol use are independent risk factors for future experiences of psychological IPV; the effect of PTSD symptoms is not conditional upon the amount of alcohol use. PTSD symptom severity, but not alcohol use, is also a risk factor for future experiences of sexual IPV. Clinically, these findings suggest that intervention efforts should explicitly target the reduction of PTSD symptoms and alcohol use problems (not necessarily concurrently) to decrease the likelihood of aggression from intimate partners. However, it is crucial that these interventions are implemented from a perspective of empowerment while promoting self-efficacy, refraining from placing blame for these types of IPV experiences on survivors' PTSD symptoms or subsequent use of alcohol to cope with their symptoms.

The current findings underscore the importance of the US Preventive Services Task Force recommendations regarding screening women of child-bearing age for past-year IPV in preventive care settings. ${ }^{80}$ Screening is often primarily thought of as a mechanism not only for identifying women who experience IPV, but it can also provide an important starting point for patient education and conversations about factors that increase risk for IPV. ${ }^{81}$ Women who experience subthreshold or probable PTSD symptoms and are heavy drinkers may benefit from tailored counseling and referrals for mental and behavioral health. Such services may reduce distress and alcohol misuse and also help mitigate risk for future IPV. Considering the findings of the present study, clinicians could use existing, brief, primary care screening measures for PTSD (e.g., Primary Care PTSD Screen for DSM-5; PC-PTSD-5), ${ }^{82}$ alcohol use (e.g., AUDIT-C), ${ }^{83}$ and $\operatorname{IPV}(e . g ., \text { HITS })^{84}$ to identify women who are at heightened risk for IPV and coordinate care accordingly with referrals to psychiatric and behavioral health services within primary care medical homes or specialty clinics.

Findings have implications for treating female veterans within VHA and non-VHA settings. Within VHA, IPV screening is recommended across primary care, mental health, substance abuse, and behavioral clinics ${ }^{85}$ where PTSD and alcohol misuse screening is also routine. Unfortunately, only $33.5 \%$ of patients with alcohol-related diagnoses are screened for IPV in comparison with $\sim 66 \%$ of patients without these diagnoses, with alcohol use predicting reduced odds of IPV screening. ${ }^{86}$ VHA providers have indicated that more psychoeducation and knowledge about the prevalence and risk factors for IPV would increase their adoption of IPV screening and counseling practices in VHA primary care. ${ }^{11,87}$ Continued dissemination of scientific evidence to providers surrounding the link between IPV and these risk factors thus remains essential, as does ensuring such screening and counseling services align with recommended best practices for delivering trauma-informed care. ${ }^{88}$

This study has several limitations. First, the participants consisted of female veterans who used VHA care, who were older, and who mainly identified as White, with no indication of their sexual orientation. As such, our findings may not generalize to non-veteran community samples, non-White female veterans, or veterans who do not use VHA care. Future research should include more racially/ethnically diverse veteran and community samples with more detailed demographic characteristics (e.g., sexual orientation) to determine if the same associations are found between PTSD and alcohol use with prospective IPV experiences. Second, the present study used self-report measures of IPV, PTSD symptoms, and alcohol use using a mail survey. Despite the strong psychometric properties of these measures, replication with semistructured diagnostic interviews, observer reports, and/or other in-person comprehensive assessments is important for corroboration of these findings. Last, there may have been response bias, in that younger women were less likely to participate in the larger study, ${ }^{45}$ making it difficult to determine if these same effects hold with more variability in age. However, given that past-year IPV, PTSD symptoms, and alcohol use are more common among younger female veterans, it is possible that the associations would be even stronger in a younger sample. ${ }^{45,89,90}$

\section{Conclusions}

This study demonstrates that the interaction of alcohol use severity and PTSD symptoms significantly heightens the risk for future physical IPV experiences. Alcohol use severity and PTSD symptom severity are also independent risk factors for sexual and psychological IPV. These findings further support the importance of informed screening practices within preventative care settings as well as mental health settings to detect those who may be at high risk for IPV as a result of PTSD symptoms and/or high severity of alcohol use problems. 


\section{Author Disclosure Statement}

No competing financial interests exist.

\section{Funding Information}

This research received financial support from the Department of Veterans Affairs, Veterans Health Administration, Health Services Research and Development (HSR\&D) Services as part of Dr. Iverson's HSR\&D Career Development Award (CDA 10-029) and Presidential Early Career Award for Scientists and Engineers (USA 14-275). Dr. Mahoney is supported by the National Institute of Mental Health award No. 5T32MH019836-16 (principal investigator: Terence Keane, $\mathrm{PhD})$.

\section{References}

1. Breiding MJ, Basile KC, Smith SG, Black MC, Mahendra RR. Intimate partner violence surveillance: Uniform definitions and recommended data elements, version 2.0. Atlanta, GA: National Center for Injury Prevention and Control, Centers for Disease Control and Prevention, 2015.

2. World Health Organization. Global and regional estimates of violence against women: Prevalence and health effects of intimate partner violence and non-partner sexual violence. World Health Organization, 2013. Available at: www.who.in Accessed May 23, 2019.

3. Smith SG, Chen J, Basile KC, et al. The National Intimate Partner and Sexual Violence Survey (NISVS): 2010-2012 state report. Atlanta, GA: National Center for Injury Prevention and Control, Centers for Disease Control and Prevention, 2017.

4. Campbell JC, Garza MA, Gielen AC, et al. Intimate partner violence and abuse among active duty military women. Violence Against Women 2003;9:1072-1092.

5. Campbell R, Greeson MR, Bybee D, Raja S. The cooccurrence of childhood sexual abuse, adult sexual assault, intimate partner violence, and sexual harassment: A mediational model of posttraumatic stress disorder and physical health outcomes. J Consult Clin Psychol 2008;76: 194-207.

6. Gerber MR, Iverson KM, Dichter ME, Klap R, Latta RE. Women veterans and intimate partner violence: Current state of knowledge and future directions. J Womens Health 2014;23:302-309.

7. Dichter ME, Cerulli C, Bossarte RM. Intimate partner violence victimization among women veterans and associated heart health risks. Womens Health Issues 2011;21:S190S194.

8. Iverson KM, Stirman SW, Street AE, et al. Female veterans' preferences for counseling related to intimate partner violence: Informing patient-centered interventions. Gen Hosp Psychiatry 2016;40:33-38.

9. Kimerling R, Iverson KM, Dichter ME, Rodriguez AL, Wong A, Pavao J. Prevalence of intimate partner violence among women veterans who utilize Veterans Health Administration primary care. J Gen Intern Med 2016;31:888-894.

10. Sherin KM, Sinacore JM, Li XQ, Zitter RE, Shakil A. HITS: A short domestic violence screening tool for use in a family practice setting. Fam Med KC 1998;30:508512.

11. Iverson KM, Vogt D, Dichter $\mathrm{ME}$, et al. Intimate partner violence and current mental health needs among female veterans. J Am Board Fam Med 2015;28:772-776.
12. Dillon G, Hussain R, Loxton D, Rahman S. Mental and physical health and intimate partner violence against women: A review of the literature. Int J Fam Med 2013; 2013:Article ID 31 3909:1-15.

13. Iverson KM, Vogt D, Maskin RM, Smith BN. Intimate partner violence victimization and associated implications for health and functioning among male and female post-9/ 11 veterans. Med Care 2017;55:S78-S84.

14. Goodman LA, Dutton MA, Vankos N, Weinfurt K. Women's resources and use of strategies as risk and protective factors for re-abuse over time. Violence Against Women 2005;11:311-336.

15. Iverson KM, Gradus JL, Resick PA, Suvak MK, Smith KF, Monson CM. Cognitive-behavioral therapy for PTSD and depression symptoms reduces risk for future intimate partner violence among interpersonal trauma survivors. J Consult Clin Psychol 2011;79:193-202.

16. Bybee DI, Sullivan CM. The process through which an advocacy intervention resulted in positive change for battered women over time. Am J Community Psychol 2002; 30:103-132.

17. Quigley BM, Leonard KE. Alcohol and the continuation of early marital aggression. Alcohol Clin Exp Res 2000;24: 1003-1010.

18. Testa M, Livingston JA, Leonard KE. Women's substance use and experiences of intimate partner violence: A longitudinal investigation among a community sample. Addict Behav 2003;28:1649-1664.

19. Cole J, Logan TK, Shannon L. Women's risk for revictimization by a new abusive partner: For what should we be looking? Violence Vict 2008;23:315-330.

20. Dietrich A. Childhood maltreatment and revictimization: The role of affect dysregulation interpersonal relatedness difficulties and posttraumatic stress disorder. J Trauma Dissociation 2007;8:25-81.

21. Gobin RL, Freyd JJ. Betrayal and revictimization: Preliminary findings. Psychol Trauma 2009;1:242-257.

22. Vatnar SKB, Bjørkly S. An interactional perspective of intimate partner violence: An in-depth semi-structured interview of a representative sample of help-seeking women. J Fam Violence 2008;23:265-279.

23. Zamir O, Lavee Y. Emotional regulation and revictimization in women's intimate relationships. J Interpers Violence 2016;31:147-162.

24. Alexander PC. Childhood trauma, attachment, and abuse by multiple partners. Psychol Trauma 2009;1:78-88.

25. Renner LM, Slack KS. Intimate partner violence and child maltreatment: Understanding intra-and intergenerational connections. Child Abuse Negl 2006;30:599-617.

26. Stein SF, Grogan-Kaylor AA, Galano MM, Clark HM, Graham-Bermann SA. The social and individual characteristics of women associated with engagement with multiple intimate violent partners. J Interpers Violence 2016: $1-25$.

27. Bogat GA, Levendosky AA, Theran S, Von Eye A, Davidson WS. Predicting the psychosocial effects of interpersonal partner violence (IPV): How much does a woman's history of IPV matter? J Interpers Violence 2003; 18:1271-1291.

28. Coolidge FL, Anderson LW. Personality profiles of women in multiple abusive relationships. J Fam Violence 2002;17: 117-131.

29. Perez S, Johnson DM. PTSD compromises battered women's future safety. J Interpers Violence 2008;23:635-651. 
30. Iverson KM, Litwack SD, Pineles SL, Suvak MK, Vaughn RA, Resick PA. Predictors of intimate partner violence revictimization: The relative impact of distinct PTSD symptoms, dissociation, and coping strategies. J Trauma Stress 2013;26:102-110.

31. Carter AC, Capone C, Short EE. Co-occurring posttraumatic stress disorder and alcohol use disorders in veteran populations. J Dual Diagn 2011;7:285-299.

32. Heltemes KJ, Clouser MC, MacGregor J, Norman MR, Galarneau MR. Co-occurring mental health and alcohol misuse: Dual disorder symptoms in combat injured veterans. Addict Behav 2010;35:840-843.

33. Norman SB, Haller M, Hamblen JL, Southwick SM, Pietrzak RH. The burden of co-occurring alcohol use disorder and PTSD in US military veterans: Comorbidities, functioning, and suicidality. Psychol Addict Behav 2018; 32:224-229.

34. Langdon KJ, Fox AB, King LA, King DW, Eisen S, Vogt D. Examination of the dynamic interplay between posttraumatic stress symptoms and alcohol misuse among combat-exposed Operation Enduring Freedom (OEF)/ Operation Iraqi Freedom (OIF) Veterans. J Affect Disord 2016;196:234-242.

35. Khantzian EJ. Understanding addictive vulnerability. Neuropsychoanalysis 2003;5:5-21.

36. Khantzian EJ. Reflections on treating addictive disorders: A psychodynamic perspective. Am J Addict 2012;21:274279.

37. Robinson J, Sareen J, Cox BJ, Bolton J. Self-medication of anxiety disorders with alcohol and drugs: Results from a nationally representative sample. J Anxiety Disord 2009; 23:38-45.

38. Waldrop AE, Back SE, Verduin ML, Brady KT. Triggers for cocaine and alcohol use in the presence and absence of posttraumatic stress disorder. Addict Behav 2007;32:634639.

39. Cohen LR, Field C, Campbell AN, Hien DA. Intimate partner violence outcomes in women with PTSD and substance use: A secondary analysis of NIDA Clinical Trials Network "Women and Trauma" Multi-site Study. Addict Behav 2013;38:2325-2332.

40. Kaysen D, Dillworth TM, Simpson T, Waldrop A, Larimer ME, Resick PA. Domestic violence and alcohol use: Trauma-related symptoms and motives for drinking. Addict Behav 2007;32:1272-1283.

41. Yalch MM, Levendosky AA. Main and moderating effects of temperament traits on the association between intimate partner violence and hazardous alcohol use in a sample of young adult women. Psychol Trauma 2018;10: 611-618.

42. Gobin RL, Green KE, Iverson KM. Alcohol misuse among female veterans: Exploring associations with interpersonal violence and mental health. Subst Use Misuse 2015;50: 1765-1777.

43. Sullivan TP, Weiss NH, Flanagan JC, Willie TC, Armeli S, Tennen H. PTSD and daily co-occurrence of drug and alcohol use among women experiencing intimate partner violence. J Dual Diagn 2016;12:36-42.

44. Reingle JM, Jennings WG, Connell NM, Businelle MS, Chartier K. On the pervasiveness of event-specific alcohol use, general substance use, and mental health problems as risk factors for intimate partner violence. J Interpers Violence 2014;29:2951-2970.
45. Louiselle M, Fuqua WR. Alcohol's effects on women's risk detection in a date-rape vignette. J Am Coll Health 2007; 55:261-266.

46. Testa M, Livingston JA. Qualitative analysis of women's experiences of sexual aggression: Focus on the role of alcohol. Psychol Women Q 1999;23:573-589.

47. Sripada CS, Angstadt M, McNamara P, King AC, Phan KL. Effects of alcohol on brain responses to social signals of threat in humans. Neuroimage 2011;55:371-380.

48. Iverson KM, Mercado R, Carpenter SL, Street AE. Intimate partner violence among women veterans: Previous interpersonal violence as a risk factor. J Trauma Stress 2013;26: 767-771.

49. Mercado RC, Wiltsey-Stirman S, Iverson KM. Impact of childhood abuse on physical and mental health status and health care utilization among female veterans. Mil Med 2015;180:1065-1074.

50. Dillman DA. Mail and internet surveys: The tailored design method. Hoboken, NJ: John Wiley and Sons, 2007.

51. McIntyre LM, Butterfield MI, Nanda K, Parsey K, Stechuchak KM, McChesney AW. Validation of a trauma questionnaire in veteran women. J Gen Intern Med 1999; 14:186-189.

52. Carlson EB, Smith SR, Palmieri PA, et al. Development and validation of a brief self-report measure of trauma exposure: The Trauma History Screen. Psychol Assess 2011;23:463-477.

53. Weathers FW, Litz BT, Herman DS, Huska JA, Keane TM. The PTSD Checklist: Reliability, validity, and diagnostic utility. Paper presented at the Annual Meeting of ISTSS, San Antonio, TX, October 1993.

54. Gore KL, McCutchan PK, Prins A, et al. Operating characteristics of the PTSD Checklist in a military primary care setting. Psychol Assess 2013;25:1032-1036.

55. Blanchard EB, Jones-Alexander J, Buckley TC, Forneris CA. Psychometric properties of the PTSD Checklist (PCL). Behav Res Ther 1996;34:669-673.

56. Ventureya V, Yao S, Cottraux J, Note I, De MeyGuillard C. The validation of the Posttraumatic Stress Disorder Checklist Scale in posttraumatic stress disorder and nonclinical subjects. Psychother Psychosom 2002; 71:47-53.

57. Ruggiero KJ, Del Ben K, Scotti JR, Rabalais AE. Psychometric properties of the PTSD Checklist-Civilian version. J Trauma Stress 2003;16:495-502.

58. Weathers FW, Litz BT, Keane TM, Palmieri PA, Marx BP, Schnurr PP. The PTSD Checklist for DSM-5 (PCL-5), 2013. Available at: www.ptsd.va.gov/professional/assessment/ adult-sr/ptsd-checklist.asp Accessed May 10, 2019.

59. Moshier SJ, Lee DJ, Bovin MJ, et al. An empirical crosswalk for the PTSD Checklist: Translating DSM-IV to DSM-5 using a veteran sample. J Trauma Stress 2019; 32:799-805. In Press.

60. Wortmann JH, Jordan AH, Weathers FW, et al. Psychometric analysis of the PTSD Checklist-5 (PCL-5) among treatment-seeking military service members. Psychol Assess 2016;28:1392-1403.

61. Bovin MJ, Marx BP, Weathers FW, et al. Psychometric properties of the PTSD Checklist for diagnostic and statistical manual of mental disorders-fifth edition (PCL-5) in veterans. Psychol Assess 2016;28:1379-1391.

62. Saunders JB, Aasland OG, Babor TF, De la Fuente JR, Grant M. Development of the Alcohol Use Disorders 
Identification Test (AUDIT): WHO collaborative project on early detection of persons with harmful alcohol consumption-II. Addict 1993;88:791-804.

63. Reinert DF, Allen JP. The Alcohol Use Disorders Identification Test (AUDIT): A review of recent research. Alcohol Clin Exp Res 2002;26:272-279.

64. Dybek I, Bischof G, Grothues J, et al. The reliability and validity of the Alcohol Use Disorders Identification Test (AUDIT) in a German general practice population sample. J Stud Alcohol 2006;67:473-481.

65. Adewuya AO. Validation of the Alcohol Use Disorders Identification Test (AUDIT) as a screening tool for alcoholrelated problems among Nigerian university students. Alcohol 2005;40;575-577.

66. Kokotailo PK, Egan J, Gangnon R, Brown D, Mundt M, Fleming M. Validity of the Alcohol Use Disorders Identification Test in college students. Alcohol Clin Exp Res 2004;28:914-920.

67. Selin KH. Test-retest reliability of the alcohol use disorder identification test in a general population sample. Alcohol Clin Exp Res 2003;27:1428-1435.

68. Straus MA, Hamby SL, Boney-McCoy S, Sugarman DB. The revised conflict tactics scales (CTS2) development and preliminary psychometric data. J Fam Issues 1996;17:283-316.

69. Coker AL, Davis KE, Arias I, et al. Physical and mental health effects of intimate partner violence for men and women. Am J Prev Med 2002;23:260-268.

70. Rabin RF, Jennings JM, Campbell JC, Bair-Merritt MH. Intimate partner violence screening tools: A systematic review. Am J Prev Med 2009;36:439-445.

71. Pico-Alfonso MA. Psychological intimate partner violence: The major predictor of posttraumatic stress disorder in abused women. Neurosci Biobehav Rev 2005;29:181-193.

72. Moffitt TE, Caspi A, Krueger RF, et al. Do partners agree about abuse in their relationship? A psychometric evaluation of interpartner agreement. Psychol Assess 1997;8:47-56.

73. Hayes AF. Introduction to mediation, moderation, and conditional process analysis: A regression-based approach. New York: Guilford Press, 2003.

74. Seal KH, Cohen G, Waldrop A, et al. Substance use disorders in Iraq and Afghanistan veterans in VA Healthcare, 2001-2010: Implications for screening, diagnosis, and treatment. Drug Alcohol Depend 2011;116:93-101.

75. Smith PH, Homish GG, Leonard KE, Cornelius JR. Intimate partner violence and specific substance use disorders: Findings from the National Epidemiologic Survey on Alcohol and Related Conditions. Psychol Addict Behav 2012;26:236-245.

76. Chavez LJ, Williams EC, Lapham G, Bradley KA. Association between alcohol screening scores and alcoholrelated risks among female Veterans Affairs patients. J Stud Alcohol Drugs 2012;73:391-400.

77. Jakupcak M, Tull MT, McDermott J, Kaysen D, Hunt S, Simpson T. PTSD symptom clusters in relationship to alcohol misuse among Iraq and Afghanistan war veterans seeking post-deployment VA health care. Addict Behav 2010;35:840-843.

78. Santana MC, Raj A, Decker MR, La Marche A, Silverman JG. Masculine gender roles associated with increased sexual risk and intimate partner violence perpetration among young adult men. J Urban Health 2006;83:575-585.
79. El-Bassel N, Gilbert L, Wu E, Go H, Hill J. Relationship between drug abuse and intimate partner violence: A longitudinal study among women receiving methadone. Am J Public Health 2005;95:465-470.

80. Curry SJ, Krist AH, Owens DK, et al. Screening for intimate partner violence, elder abuse, and abuse of vulnerable adults: US Preventive Services Task Force final recommendation statement. JAMA 2018;320:1678-1687.

81. Iverson KM., Adjognon $\mathrm{O}$, Grillo $\mathrm{AR}$, et al. Intimate partner violence screening programs in the Veterans Health Administration: Informing scale-up of successful practices. J Gen Intern Med 2019;34:2435-2442. In Press.

82. Prins A, Bovin MJ, Smolinski DJ, et al. The Primary Care PTSD Screen for DSM-5 (PC-PTSD-5): Development and evaluation within a veteran primary care sample. J Gen Intern Med; 31:1206-1211.

83. Bradley KA, DeBenedetti AF, Volk RJ, Williams EC, Frank D, Kivlahan DR. AUDIT-C as a brief screen for alcohol misuse in primary care. Alcohol Clin Exp Res 2007;31:1208-1217.

84. Iverson KM, King MW, Resick PA, Gerber MR, Kimerling R, Vogt D. Clinical utility of an intimate partner violence screening tool for female VHA patients. J Gen Intern Med 2013;28:1288-1293.

85. Veterans Health Administration. Intimate partner violence assistance program. Washington, DC: Department of Veterans Affairs, Health Administration, 2019.

86. Choo EK, Nicolaidis C, Jenkinson RH, Cox JM, McConnell KJ. Failure of intimate partner violence screening among patients with substance use disorders. Acad Emerg Med 2010;17:886-889.

87. Iverson KM, Wells SY, Wiltsey-Stirman S, Vaughn R, Gerber MR. VHA primary care providers' perspectives on screening female veterans for intimate partner violence: A preliminary assessment. J Fam Violence 2013; 28:823-831.

88. Gerber MR. Trauma-Informed Healthcare Approaches: A Guide for Primary Care. New York, NY: Springer; 2019.

89. Smith BN, Tyzik AL, Iverson KM. Age-related differences in trauma exposure, PTSD symptomatology, and functional health and well-being in women veterans. Traumatology 2015;21:128-135.

90. Frayne SM, Phibbs CS, Saechao F, et al. Sourcebook: Women veterans in the Veterans Health Administration. Volume 4: Longitudinal trends in sociodemographics, utilization, health profile, and geographic distribution. Washington, DC: Women's Health Evaluation Initiative, Women's Health Services, Veterans Health Administration, Department of Veterans Affairs, February 2018.

Address correspondence to: Colin T. Mahoney, PhD Behavioral Science Division National Center for PTSD VA Boston Healthcare System 150 S. Huntington Avenue (116B-4) Boston, MA 02130

E-mail: colintmahoney@gmail.com 